S2 Table. MV by gender and ethnicity, $95 \% \mathrm{CI}$, results of $\chi^{2}$ tests and residual analysis

The prevalence of lifetime experience of different types of marital violence by gender and ethnicity

\begin{tabular}{|c|c|c|c|c|c|c|c|c|c|c|c|c|}
\hline & \multicolumn{4}{|c|}{ Overall } & \multicolumn{4}{|c|}{ Men } & \multicolumn{4}{|c|}{ Women } \\
\hline & Total & Garo & Santal & Bengali & Total & Garo & Santal & Bengali & Total & Garo & Santal & Bengali \\
\hline & $\begin{array}{r}\mathrm{N}=1929(\%) \\
(95 \% \mathrm{Cl}) \\
\end{array}$ & $\begin{array}{r}\mathrm{N}=640(\%) \\
(95 \% \mathrm{Cl}) \\
\end{array}$ & $\begin{array}{r}\mathrm{N}=640(\%) \\
(95 \% \mathrm{Cl})\end{array}$ & $\begin{array}{r}\mathrm{N}=649(\%) \\
(95 \% \mathrm{Cl}) \\
\end{array}$ & $\begin{array}{r}\mathrm{n}=960(\%) \\
(95 \% \mathrm{Cl}) \\
\end{array}$ & $\begin{array}{r}\mathrm{n}=318(\%) \\
(95 \% \mathrm{Cl})\end{array}$ & $\begin{array}{r}n=319(\%) \\
(95 \% \mathrm{Cl}) \\
\end{array}$ & $\begin{array}{r}n=323(\%) \\
(95 \% \mathrm{Cl})\end{array}$ & $\begin{array}{r}\mathrm{n}=969(\%) \\
(95 \% \mathrm{Cl}) \\
\end{array}$ & $\begin{array}{r}n=322(\%) \\
(95 \% \mathrm{Cl})\end{array}$ & $\begin{array}{r}\mathrm{n}=321(\%) \\
(95 \% \mathrm{Cl}) \\
\end{array}$ & $\begin{array}{r}n=326(\%) \\
(95 \% \mathrm{Cl})\end{array}$ \\
\hline \multicolumn{13}{|l|}{ Emotional violence } \\
\hline No & $\begin{array}{r}910(47.2) \\
(45.0-49.4)\end{array}$ & \begin{tabular}{|r|}
$297(46.4)$ \\
$(42.8-50.3)$ \\
\end{tabular} & $\begin{array}{c}307(48.0) \\
(44.1-51.7)\end{array}$ & $\begin{array}{r}306(47.1) \\
(43.3-51.2) \\
\end{array}$ & $\begin{array}{r}867(90.3) \\
(88.3-92.2) \\
\end{array}$ & $\begin{array}{r}267(84.0) \\
(79.6-87.4) \\
\end{array}$ & $\begin{array}{r}305(95.6) \\
(93.1-97.8) \\
\end{array}$ & $\begin{array}{r}295(91.3) \\
(88.2-94.4)\end{array}$ & $\begin{array}{r}43(4.4) \\
(3.2-5.8) \\
\end{array}$ & $\begin{array}{r}30(9.3) \\
(6.2-12.7) \\
\end{array}$ & $\begin{array}{r}2(0.6) \\
(0.0-1.6) \\
\end{array}$ & $\begin{array}{r}11(3.4) \\
(1.5-5.5)\end{array}$ \\
\hline Yes & $\begin{array}{r}1019(52.8) \\
(50.6-55.0) \\
\end{array}$ & $\begin{array}{r}343(53.6) \\
(49.7-57.2) \\
\end{array}$ & $\begin{array}{c}333(52.0) \\
(48.3-55.9) \\
\end{array}$ & $\begin{array}{r}343(52.9) \\
(48.8-56.7) \\
\end{array}$ & $\begin{array}{r}93(9.7) \\
(7.8-11.7) \\
\end{array}$ & $\begin{array}{r}51(16.0) \\
(12.6-20.4) \\
\end{array}$ & $\begin{array}{r}14(4.4) \\
(2.2-6.9) \\
\end{array}$ & $\begin{array}{r}28(8.7) \\
(5.6-11.8) \\
\end{array}$ & $\begin{array}{r}926(95.6) \\
(94.2-96.8) \\
\end{array}$ & $\begin{array}{r}292(90.7) \\
(87.3-93.8) \\
\end{array}$ & $\begin{array}{r}319(99.4) \\
(98.4-100.0) \\
\end{array}$ & $\begin{array}{r}315(96.6) \\
(94.5-98.5) \\
\end{array}$ \\
\hline Mild & $\begin{array}{r}431(22.3) \\
(20.5-24.1)\end{array}$ & \begin{tabular}{|r|}
$182(28.4)$ \\
$(25.0-31.9)$ \\
\end{tabular} & $\begin{array}{c}136(21.3) \\
(18.1-24.4)\end{array}$ & $\begin{array}{r}113(17.4) \\
(14.5-20.5)\end{array}$ & $\begin{array}{r}61(6.4) \\
(4.8-7.9)\end{array}$ & $\begin{array}{r}32(10.1) \\
(7.2-13.5)\end{array}$ & $\begin{array}{r}12(3.8) \\
(1.9-6.0) \\
\end{array}$ & $\begin{array}{r}17(5.3) \\
(2.8-7.7) \\
\end{array}$ & $\begin{array}{r}370(38.2) \\
(35.1-41.3) \\
\end{array}$ & $\begin{array}{r}150(46.6) \\
(41.0-52.2) \\
\end{array}$ & $\begin{array}{r}124(38.6) \\
(33.3-44.2) \\
\end{array}$ & $\begin{array}{r}96(29.4) \\
(25.2-34.4) \\
\end{array}$ \\
\hline Severe & $\begin{array}{r}588(30.5) \\
(28.4-32.5) \\
\end{array}$ & $\begin{array}{r}161(25.2) \\
(22.0-28.6) \\
\end{array}$ & $\begin{array}{c}197(30.8) \\
(27.3-34.5)\end{array}$ & $\begin{array}{r}230(35.4) \\
(31.7-39.1) \\
\end{array}$ & $\begin{array}{r}32(3.3) \\
(2.2-4.6) \\
\end{array}$ & $\begin{array}{r}19(6.0) \\
(3.5-8.8) \\
\end{array}$ & $\begin{array}{r}2(0.6) \\
(0.0-1.6) \\
\end{array}$ & $\begin{array}{l}11(03.4) \\
(1.5-5.6) \\
\end{array}$ & $\begin{array}{r}556(57.4) \\
(54.3-60.8) \\
\end{array}$ & $\begin{array}{r}142(44.1) \\
(38.5-49.4) \\
\end{array}$ & $\begin{array}{r}195(60.7) \\
(55.1-66.0) \\
\end{array}$ & $\begin{array}{r}219(67.2) \\
(62.0-71.8) \\
\end{array}$ \\
\hline \multicolumn{13}{|l|}{ Physical violence } \\
\hline No & $\begin{array}{l}1307(67.8) \\
(65.6-70.0) \\
\end{array}$ & $\begin{array}{r}463(72.3) \\
(68.9-75.6) \\
\end{array}$ & $\begin{array}{c}423(66.1) \\
(62.2-69.8) \\
\end{array}$ & $\begin{array}{r}421(64.9) \\
(61.3-68.6) \\
\end{array}$ & $\begin{array}{r}953(99.3) \\
(98.6-99.8) \\
\end{array}$ & $\begin{array}{r}313(98.4) \\
(96.9-99.7) \\
\end{array}$ & $\begin{array}{r}318(99.7) \\
(98.7-100.0) \\
\end{array}$ & $\begin{array}{r}322(99.7) \\
(99.1-100.0) \\
\end{array}$ & $\begin{array}{r}354(36.5) \\
(33.5-39.6) \\
\end{array}$ & $\begin{array}{r}150(46.6) \\
(41.3-52.5) \\
\end{array}$ & $\begin{array}{r}105(32.7) \\
(27.7-37.7) \\
\end{array}$ & $\begin{array}{r}99(30.4) \\
(25.5-35.6) \\
\end{array}$ \\
\hline Yes & $\begin{array}{r}622(32.2) \\
(30.0-34.4) \\
\end{array}$ & \begin{tabular}{|r|}
$177(27.7)$ \\
$(24.4-31.1)$ \\
\end{tabular} & $\begin{array}{c}217(33.9) \\
(30.2-37.8) \\
\end{array}$ & $\begin{array}{r}228(35.1) \\
(31.4-38.7) \\
\end{array}$ & $\begin{array}{r}7(0.7) \\
(0.2-1.4) \\
\end{array}$ & $\begin{array}{r}5(1.6) \\
(0.3-3.1) \\
\end{array}$ & $\begin{array}{r}1(0.3) \\
(0.0-1.3) \\
\end{array}$ & $\begin{array}{r}1(0.3) \\
(0.0-0.9) \\
\end{array}$ & $\begin{array}{r}615(63.5) \\
(60.4-66.5) \\
\end{array}$ & $\begin{array}{r}172(53.4) \\
(47.5-58.7) \\
\end{array}$ & $\begin{array}{r}216(67.3) \\
(62.3-72.3) \\
\end{array}$ & $\begin{array}{r}227(69.6) \\
(64.4-74.5) \\
\end{array}$ \\
\hline Mild & $\begin{array}{r}281(14.6) \\
(13.0-16.1)\end{array}$ & $\begin{array}{r}70(10.9) \\
(8.4-13.6) \\
\end{array}$ & $\begin{array}{r}99(15.5) \\
(12.8-18.3)\end{array}$ & $\begin{array}{r}112(17.3) \\
(14.3-20.3)\end{array}$ & $\begin{array}{r}5(0.5) \\
(0.1-1.0) \\
\end{array}$ & $\begin{array}{r}3(0.9) \\
(0.0-2.2)\end{array}$ & $\begin{array}{r}1(0.3) \\
(0.0-1.3) \\
\end{array}$ & $\begin{array}{r}1(0.3) \\
(0.0-0.9)\end{array}$ & $\begin{array}{r}276(28.5) \\
(25.7-31.3) \\
\end{array}$ & $\begin{array}{r}67(20.8) \\
(16.5-25.2) \\
\end{array}$ & $\begin{array}{r}98(30.5) \\
(25.9-35.5) \\
\end{array}$ & $\begin{array}{r}111(34.0) \\
(29.1-39.0)\end{array}$ \\
\hline Severe & $\begin{array}{r}341(17.7) \\
(16.0-19.2)\end{array}$ & \begin{tabular}{|r|}
$107(16.7)$ \\
$(13.8-19.8)$ \\
\end{tabular} & $\begin{array}{c}118(18.4) \\
(15.5-21.4)\end{array}$ & $\begin{array}{r}116(17.9) \\
(14.8-20.8)\end{array}$ & $\begin{array}{r}2(0.2) \\
(0.0-0.5) \\
\end{array}$ & $\begin{array}{r}2(0.6) \\
(0.0-1.6) \\
\end{array}$ & $\begin{array}{r}0(0.0) \\
(0.0-0.0) \\
\end{array}$ & $\begin{array}{r}0(0.0) \\
(0.0-0.0)\end{array}$ & $\begin{array}{r}339(35.0) \\
(32.1-38.1) \\
\end{array}$ & $\begin{array}{r}105(32.6) \\
(27.6-38.2) \\
\end{array}$ & $\begin{array}{r}118(36.8) \\
(31.8-41.7) \\
\end{array}$ & $\begin{array}{r}116(35.6) \\
(30.4-40.8)\end{array}$ \\
\hline \multicolumn{13}{|l|}{ Sexual violence } \\
\hline No & $\begin{array}{l}1236(64.1) \\
(62.1-66.2)\end{array}$ & $\begin{array}{r}434(67.8) \\
(64.2-71.4) \\
\end{array}$ & $\begin{array}{c}411(64.2) \\
(60.6-67.8)\end{array}$ & $\begin{array}{r}391(60.2) \\
(56.4-63.9)\end{array}$ & $\begin{array}{r}959(99.9) \\
(99.6-100.0) \\
\end{array}$ & $\begin{array}{r}318(100.0) \\
(100.0-100.0) \\
\end{array}$ & $\begin{array}{r}319(100.0) \\
(100.0-100.0) \\
\end{array}$ & $\begin{array}{r}322(99.7) \\
(99.1-100.0) \\
\end{array}$ & $\begin{array}{r}277(28.6) \\
(25.6-31.5) \\
\end{array}$ & $\begin{array}{r}116(36.0) \\
(31.1-41.6)\end{array}$ & $\begin{array}{r}92(28.7) \\
(23.7-33.6) \\
\end{array}$ & $\begin{array}{r}69(21.2) \\
(17.2-25.5) \\
\end{array}$ \\
\hline Yes & $\begin{array}{r}693(35.9) \\
(33.8-37.9)\end{array}$ & \begin{tabular}{|r|}
$206(32.2)$ \\
$(28.6-35.8)$
\end{tabular} & $\begin{array}{c}229(35.8) \\
(32.2-39.4)\end{array}$ & $\begin{array}{r}258(39.8) \\
(36.1-43.6)\end{array}$ & $\begin{array}{r}1(0.1) \\
(0.0-0.4)\end{array}$ & $\begin{array}{r}0(0.0) \\
(0.0-0.0)\end{array}$ & $\begin{array}{r}0(0.0) \\
(0.0-0.0)\end{array}$ & $\begin{array}{r}1(0.3) \\
(0.0-0.9)\end{array}$ & $\begin{array}{r}692(71.4) \\
(68.5-74.4)\end{array}$ & $\begin{array}{r}206(64.0) \\
(58.4-68.9)\end{array}$ & $\begin{array}{r}229(71.3) \\
(66.4-76.3)\end{array}$ & $\begin{array}{r}257(78.8) \\
(74.5-82.8)\end{array}$ \\
\hline Mild & $\begin{array}{r}63(3.3) \\
(2.4-4.0) \\
\end{array}$ & $\begin{array}{r}22(3.4) \\
(2.0-5.0) \\
\end{array}$ & $\begin{array}{c}20(3.1) \\
(1.9-4.5) \\
\end{array}$ & $\begin{array}{r}21(3.2) \\
(1.8-4.6) \\
\end{array}$ & $\begin{array}{r}1(00.1) \\
(0.0-0.4) \\
\end{array}$ & $\begin{array}{r}0(0.0) \\
(0.0-0.0) \\
\end{array}$ & $\begin{array}{r}0(0.0) \\
(0.0-0.0) \\
\end{array}$ & $\begin{array}{r}1(0.3) \\
(0.0-0.9) \\
\end{array}$ & $\begin{array}{r}62(6.4) \\
(4.9-8.0) \\
\end{array}$ & $\begin{array}{r}22(6.8) \\
(4.0-9.6) \\
\end{array}$ & $\begin{array}{r}20(6.2) \\
(3.7-9.0) \\
\end{array}$ & $\begin{array}{r}20(6.1) \\
(3.7-8.9) \\
\end{array}$ \\
\hline Severe & $\begin{array}{r}630(32.7) \\
(30.6-34.6) \\
\end{array}$ & \begin{tabular}{|r|}
$184(28.7)$ \\
$(25.3-32.3)$ \\
\end{tabular} & $\begin{array}{c}209(32.7) \\
(29.2-36.1)\end{array}$ & $\begin{array}{r}237(36.5) \\
(33.0-40.4)\end{array}$ & $\begin{array}{r}0(0.0) \\
(0.0-0.0) \\
\end{array}$ & $\begin{array}{r}0(0.0) \\
(0.0-0.0) \\
\end{array}$ & $\begin{array}{r}0(0.0) \\
(0.0-0.0) \\
\end{array}$ & $\begin{array}{r}0(0.0) \\
(0.0-0.0)\end{array}$ & $\begin{array}{r}630(65.0) \\
(61.9-68.0) \\
\end{array}$ & $\begin{array}{r}184(57.1) \\
(51.6-62.4) \\
\end{array}$ & $\begin{array}{r}209(65.1) \\
(59.8-70.4) \\
\end{array}$ & $\begin{array}{r}237(72.7) \\
(67.5-77.3)\end{array}$ \\
\hline \multicolumn{13}{|l|}{ Overall violence } \\
\hline Any type (yes) & $\begin{array}{r}1033(53.6) \\
(51.3-55.7) \\
\end{array}$ & \begin{tabular}{|r|}
$351(54.8)$ \\
$(50.9-58.4)$ \\
\end{tabular} & $\begin{array}{c}333(52.0) \\
(48.3-55.9)\end{array}$ & $\begin{array}{r}349(53.8) \\
(49.9-57.6)\end{array}$ & $\begin{array}{r}95(9.9) \\
(8.0-11.9) \\
\end{array}$ & $\begin{array}{r}52(16.4) \\
(12.9-20.8) \\
\end{array}$ & $\begin{array}{r}14(4.4) \\
(2.2-6.9) \\
\end{array}$ & $\begin{array}{r}29(9.0) \\
(5.9-12.1)\end{array}$ & $\begin{array}{r}938(96.8) \\
(95.8-97.8) \\
\end{array}$ & $\begin{array}{r}299(92.9) \\
(89.8-95.3) \\
\end{array}$ & \begin{tabular}{|r|}
$319(99.4)$ \\
$(98.4-100.0)$ \\
\end{tabular} & $\begin{array}{r}320(98.2) \\
(96.3-99.4)\end{array}$ \\
\hline \begin{tabular}{|l|} 
Poly abuse (yes) \\
\end{tabular} & $\begin{array}{r}490(25.4) \\
(23.4-27.2) \\
\end{array}$ & \begin{tabular}{|r|}
$125(19.5)$ \\
$(16.6-22.7)$ \\
\end{tabular} & $\begin{array}{c}173(27.0) \\
(23.6-30.5)\end{array}$ & $\begin{array}{r}192(29.6) \\
(26.2-33.0)\end{array}$ & $\begin{array}{r}0(0.0) \\
(0.0-0.0) \\
\end{array}$ & $\begin{array}{r}0(0.0) \\
(0.0-0.0)\end{array}$ & $\begin{array}{r}0(0.0) \\
(0.0-0.0)\end{array}$ & $\begin{array}{r}0(0.0) \\
(0.0-0.0)\end{array}$ & $\begin{array}{r}490(50.6) \\
(47.4-53.4)\end{array}$ & $\begin{array}{r}125(38.8) \\
(33.5-44.1) \\
\end{array}$ & $\begin{array}{r}173(53.9) \\
(48.9-59.2) \\
\end{array}$ & $\begin{array}{r}192(58.9) \\
(53.4-64.1) \\
\end{array}$ \\
\hline
\end{tabular}

Note: $95 \% \mathrm{Cl}$ bootstrap results are based on 1000 bootstrap samples 


\section{Results of $\chi^{2}$ tests and post hoc (residual) analysis}

\section{Experience of MV by ethnicity}

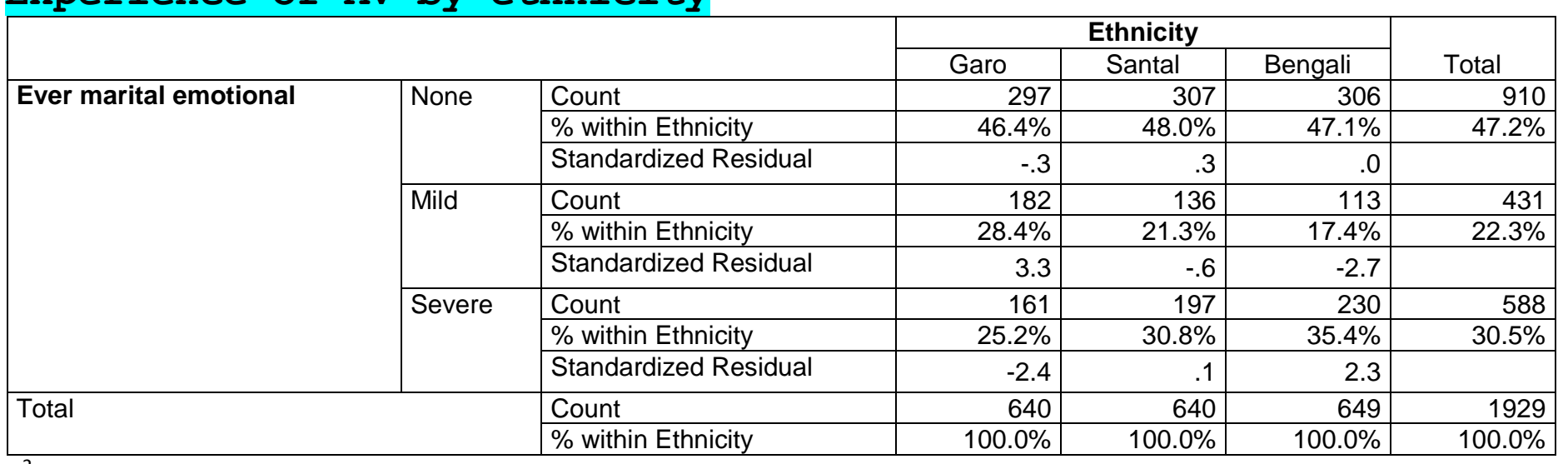

$\chi^{2}=29.41, \mathrm{df}=4, \mathrm{p}<.001$

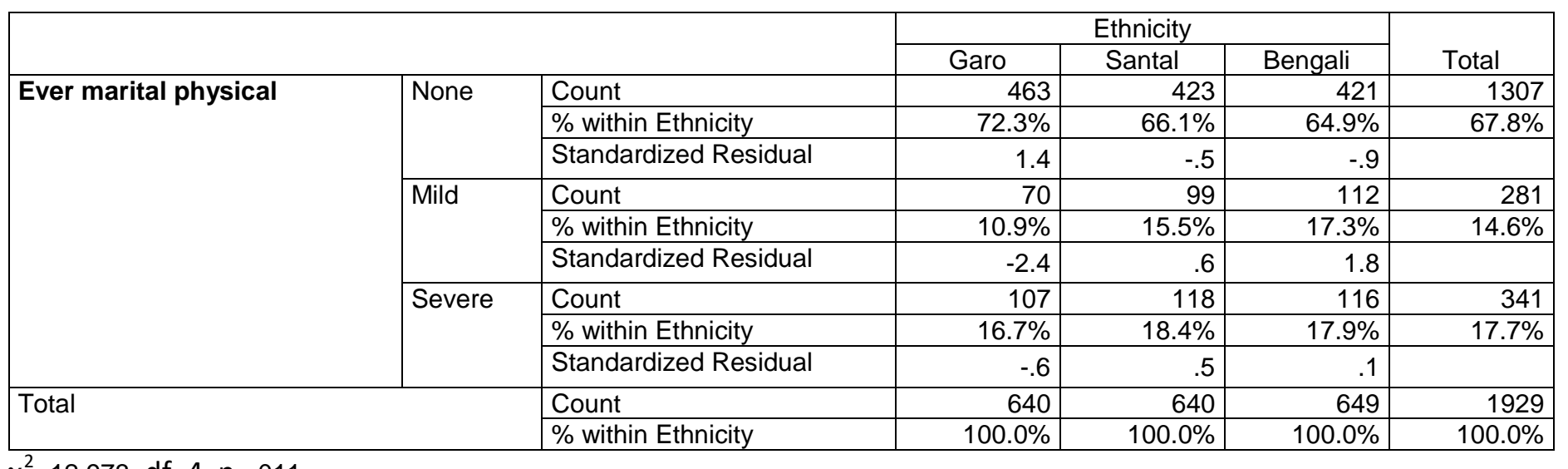

$\chi^{2}=12.973, d f=4, p=.011$ 


\begin{tabular}{|c|c|c|c|c|c|c|}
\hline & & & \multicolumn{3}{|c|}{ Ethnicity } & \multirow[b]{2}{*}{ Total } \\
\hline & & & Garo & Santal & Bengali & \\
\hline \multirow[t]{9}{*}{ Ever severe sexual severity } & \multirow[t]{3}{*}{ None } & Count & 434 & 411 & 391 & 1236 \\
\hline & & \% within Ethnicity & $67.8 \%$ & $64.2 \%$ & $60.2 \%$ & $64.1 \%$ \\
\hline & & Standardized Residual & 1.2 & .0 & -1.2 & \\
\hline & \multirow[t]{3}{*}{ Mild } & Count & 22 & 20 & 21 & 63 \\
\hline & & \% within Ethnicity & $3.4 \%$ & $3.1 \%$ & $3.2 \%$ & $3.3 \%$ \\
\hline & & Standardized Residual & .2 & -.2 & .0 & \\
\hline & \multirow[t]{3}{*}{ Severe } & Count & 184 & 209 & 237 & 630 \\
\hline & & $\%$ within Ethnicity & $28.7 \%$ & $32.7 \%$ & $36.5 \%$ & $32.7 \%$ \\
\hline & & Standardized Residual & -1.7 & .0 & 1.7 & \\
\hline \multirow{2}{*}{\multicolumn{2}{|c|}{ Total }} & Count & 640 & 640 & 649 & 1929 \\
\hline & & \% within Ethnicity & $100.0 \%$ & $100.0 \%$ & $100.0 \%$ & $100.0 \%$ \\
\hline
\end{tabular}

$\chi^{2}=8.934, d f=4, p=.063$

\begin{tabular}{|c|c|c|c|c|c|c|}
\hline & & & & Ethnicity & & \\
\hline & & & Garo & Santal & Bengali & Total \\
\hline Ever marital any abuse & No & Count & 289 & 307 & 300 & 896 \\
\hline & & \% within Ethnicity & $45.2 \%$ & $48.0 \%$ & $46.2 \%$ & $46.4 \%$ \\
\hline & & Standardized Residual & -.5 & .6 & -.1 & \\
\hline & Yes & Count & 351 & 333 & 349 & 1033 \\
\hline & & $\%$ within Ethnicity & $54.8 \%$ & $52.0 \%$ & $53.8 \%$ & $53.6 \%$ \\
\hline & & Standardized Residual & .4 & -.5 & .1 & \\
\hline Total & & Count & 640 & 640 & 649 & 1929 \\
\hline & & \% within Ethnicity & $100.0 \%$ & $100.0 \%$ & $100.0 \%$ & $100.0 \%$ \\
\hline
\end{tabular}

$\chi^{2}=1.037, d f=2, p=.595$

\begin{tabular}{|c|c|c|c|c|c|c|}
\hline & \multicolumn{3}{|c|}{ Ethnicity } & \multirow[b]{2}{*}{ Total } \\
\hline & & & Garo & Santal & Bengali & \\
\hline \multirow[t]{6}{*}{ Ever marital poly abuse } & \multirow[t]{3}{*}{ No } & Count & 515 & 467 & 457 & 1439 \\
\hline & & \% within Ethnicity & $80.5 \%$ & $73.0 \%$ & $70.4 \%$ & $74.6 \%$ \\
\hline & & Standardized Residual & 1.7 & -.5 & -1.2 & \\
\hline & \multirow[t]{3}{*}{ Yes } & Count & 125 & 173 & 192 & 490 \\
\hline & & $\%$ within Ethnicity & $19.5 \%$ & $27.0 \%$ & $29.6 \%$ & $25.4 \%$ \\
\hline & & Standardized Residual & -2.9 & .8 & 2.1 & \\
\hline \multirow{2}{*}{\multicolumn{2}{|c|}{ Total }} & Count & 640 & 640 & 649 & 1929 \\
\hline & & \% within Ethnicity & $100.0 \%$ & $100.0 \%$ & $100.0 \%$ & $100.0 \%$ \\
\hline
\end{tabular}

$\chi^{2}=18.527, d f=2, p<.001$ 
Experience of MV among women by ethnicity

\begin{tabular}{|c|c|c|c|c|c|c|}
\hline & \multicolumn{3}{|c|}{ Ethnicity } & \multirow[b]{2}{*}{ Total } \\
\hline & & & Garo & Santal & Bengali & \\
\hline \multirow{9}{*}{ Ever marital emotional severity } & \multirow{3}{*}{ None } & Count & 30 & 2 & 11 & 43 \\
\hline & & \% within Ethnicity & $9.3 \%$ & $0.6 \%$ & $3.4 \%$ & $4.4 \%$ \\
\hline & & Standardized Residual & 4.2 & -3.2 & -.9 & \\
\hline & \multirow{3}{*}{ Mild } & Count & 150 & 124 & 96 & 370 \\
\hline & & \% within Ethnicity & $46.6 \%$ & $38.6 \%$ & $29.4 \%$ & $38.2 \%$ \\
\hline & & Standardized Residual & 2.4 & .1 & -2.6 & \\
\hline & \multirow{3}{*}{ Severe } & Count & 142 & 195 & 219 & 556 \\
\hline & & $\%$ within Ethnicity & $44.1 \%$ & $60.7 \%$ & $67.2 \%$ & $57.4 \%$ \\
\hline & & Standardized Residual & -3.1 & .8 & 2.3 & \\
\hline \multirow{2}{*}{\multicolumn{2}{|c|}{ Total }} & Count & 322 & 321 & 326 & 969 \\
\hline & & \% within Ethnicity & $100.0 \%$ & $100.0 \%$ & $100.0 \%$ & $100.0 \%$ \\
\hline
\end{tabular}

$\chi^{2}=57.100, d f=4, p<.001$

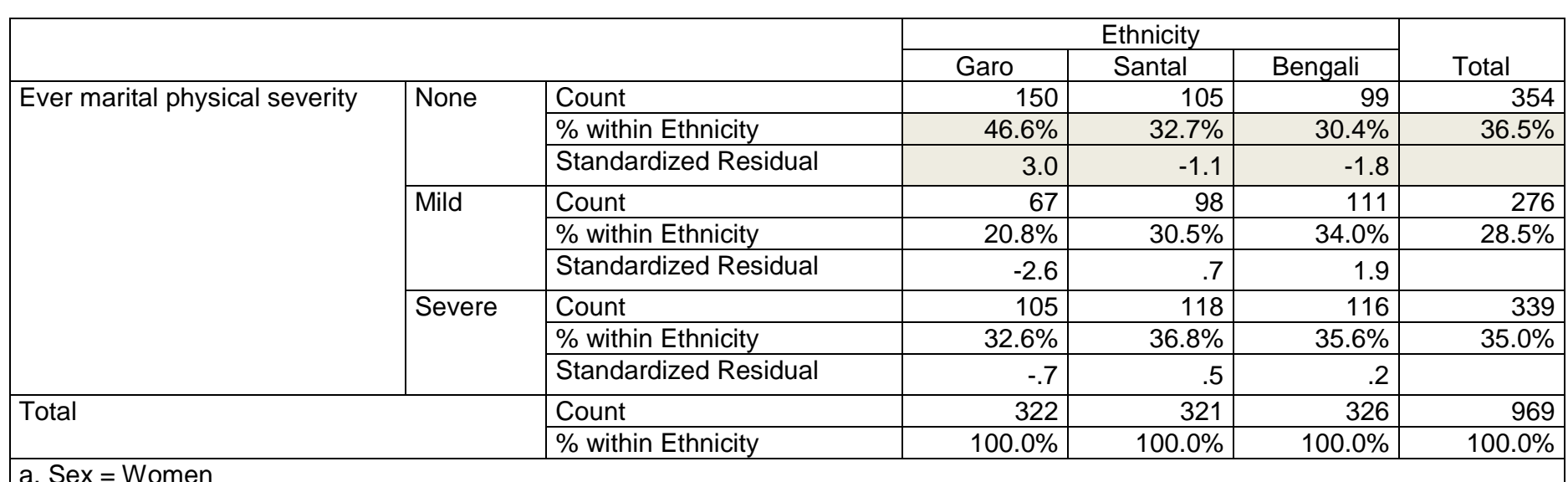

$\chi^{2}=25.100, d f=4, p<.001$ 


\begin{tabular}{|c|c|c|c|c|c|c|}
\hline & & & \multicolumn{3}{|c|}{ Ethnicity } & \multirow[b]{2}{*}{ Total } \\
\hline & & & Garo & Santal & Bengali & \\
\hline \multirow[t]{9}{*}{ Ever severe sexual severity } & \multirow[t]{3}{*}{ None } & Count & 116 & 92 & 69 & 277 \\
\hline & & \% within Ethnicity & $36.0 \%$ & $28.7 \%$ & $21.2 \%$ & $28.6 \%$ \\
\hline & & Standardized Residual & 2.5 & .0 & -2.5 & \\
\hline & \multirow[t]{3}{*}{ Mild } & Count & 22 & 20 & 20 & 62 \\
\hline & & $\%$ within Ethnicity & $6.8 \%$ & $6.2 \%$ & $6.1 \%$ & $6.4 \%$ \\
\hline & & Standardized Residual & .3 & -.1 & -.2 & \\
\hline & \multirow[t]{3}{*}{ Severe } & Count & 184 & 209 & 237 & 630 \\
\hline & & \% within Ethnicity & $57.1 \%$ & $65.1 \%$ & $72.7 \%$ & $65.0 \%$ \\
\hline & & Standardized Residual & -1.8 & .0 & 1.7 & \\
\hline \multirow{2}{*}{\multicolumn{2}{|c|}{ Total }} & Count & 322 & 321 & 326 & 969 \\
\hline & & \% within Ethnicity & $100.0 \%$ & $100.0 \%$ & $100.0 \%$ & $100.0 \%$ \\
\hline
\end{tabular}

$\chi^{2}=18.688, d f=4, p=.001$

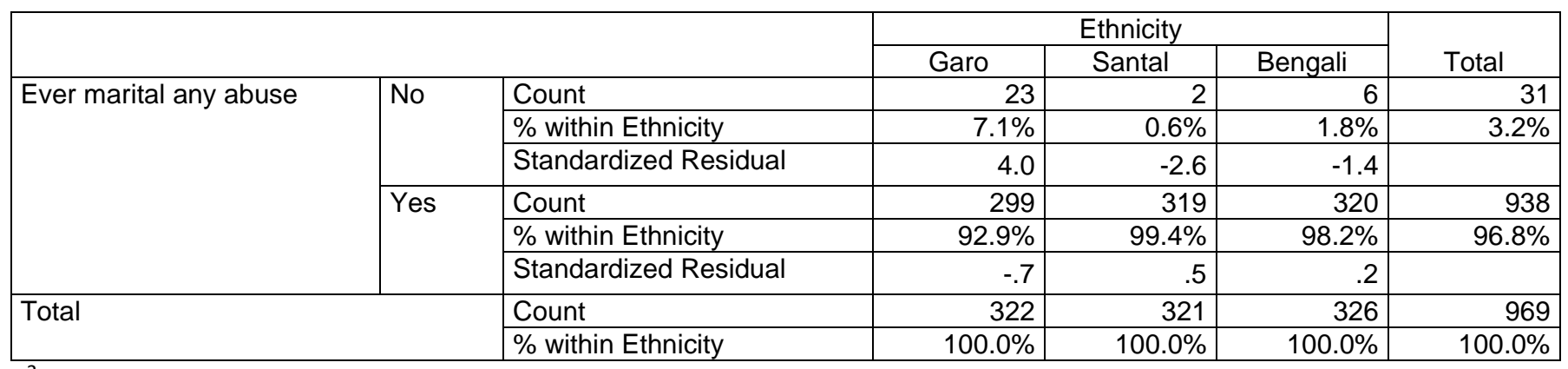

$\chi^{2}=24.993, \mathrm{df}=2,<=.001$

\begin{tabular}{|c|c|c|c|c|c|c|}
\hline & \multicolumn{3}{|c|}{ Ethnicity } & \multirow[b]{2}{*}{ Total } \\
\hline & & & Garo & Santal & Bengali & \\
\hline \multirow[t]{6}{*}{ Ever marital poly abuse } & \multirow[t]{3}{*}{ No } & Count & 197 & 148 & 134 & 479 \\
\hline & & $\%$ within Ethnicity & $61.2 \%$ & $46.1 \%$ & $41.1 \%$ & $49.4 \%$ \\
\hline & & Standardized Residual & 3.0 & -.8 & -2.1 & \\
\hline & \multirow[t]{3}{*}{ Yes } & Count & 125 & 173 & 192 & 490 \\
\hline & & \% within Ethnicity & $38.8 \%$ & $53.9 \%$ & $58.9 \%$ & $50.6 \%$ \\
\hline & & Standardized Residual & -3.0 & .8 & 2.1 & \\
\hline \multirow{2}{*}{\multicolumn{2}{|c|}{ Total }} & Count & 322 & 321 & 326 & 969 \\
\hline & & \% within Ethnicity & $100.0 \%$ & $100.0 \%$ & $100.0 \%$ & $100.0 \%$ \\
\hline
\end{tabular}

$\chi^{2}=28.244, \mathrm{df}=2,<=.001$ 


\section{Experience of MV among men by ethnicity}

\begin{tabular}{|c|c|c|c|c|c|c|}
\hline & & & \multicolumn{3}{|c|}{ Ethnicity } & \multirow[b]{2}{*}{ Total } \\
\hline & & & Garo & Santal & Bengali & \\
\hline \multirow[t]{6}{*}{ Ever marital emotional severity } & \multirow[t]{2}{*}{ None } & Count & 267 & 305 & 295 & 867 \\
\hline & & \% within Ethnicity & $84.0 \%$ & $95.6 \%$ & $91.3 \%$ & $90.3 \%$ \\
\hline & \multirow[t]{2}{*}{ Mild } & Count & 32 & 12 & 17 & 61 \\
\hline & & \% within Ethnicity & $10.1 \%$ & $3.8 \%$ & $5.3 \%$ & $6.4 \%$ \\
\hline & \multirow{2}{*}{ Severe } & \% within Ethnicity & $6.0 \%$ & $0.6 \%$ & $3.4 \%$ & $3.3 \%$ \\
\hline & & Standardized Residual & 2.6 & -2.6 & .1 & \\
\hline \multirow{2}{*}{\multicolumn{2}{|c|}{ Total }} & Count & 318 & 319 & 323 & 960 \\
\hline & & \% within Ethnicity & $100.0 \%$ & $100.0 \%$ & $100.0 \%$ & $100.0 \%$ \\
\hline
\end{tabular}

$\chi^{2}=26.983, \mathrm{df}=4, \mathrm{p}<.001$

\begin{tabular}{|c|c|c|c|c|c|c|}
\hline \multicolumn{7}{|c|}{ Crosstab $^{a}$} \\
\hline & & & \multicolumn{3}{|c|}{ Ethnicity } & \multirow[b]{2}{*}{ Total } \\
\hline & & & Garo & Santal & Bengali & \\
\hline \multirow[t]{9}{*}{ Ever marital physical severity } & \multirow[t]{3}{*}{ None } & Count & 313 & 318 & 322 & 953 \\
\hline & & $\%$ within Ethnicity & $98.4 \%$ & $99.7 \%$ & $99.7 \%$ & $99.3 \%$ \\
\hline & & Standardized Residual & -.2 & .1 & .1 & \\
\hline & \multirow[t]{3}{*}{ Mild } & Count & 3 & 1 & 1 & 5 \\
\hline & & $\%$ within Ethnicity & $0.9 \%$ & $0.3 \%$ & $0.3 \%$ & $0.5 \%$ \\
\hline & & Standardized Residual & 1.0 & -.5 & -.5 & \\
\hline & \multirow[t]{3}{*}{ Severe } & Count & 2 & 0 & 0 & 2 \\
\hline & & $\%$ within Ethnicity & $0.6 \%$ & $0.0 \%$ & $0.0 \%$ & $0.2 \%$ \\
\hline & & Standardized Residual & 1.6 & -.8 & -.8 & \\
\hline \multirow{2}{*}{\multicolumn{2}{|c|}{ Total }} & Count & 318 & 319 & 323 & 960 \\
\hline & & \% within Ethnicity & $100.0 \%$ & $100.0 \%$ & $100.0 \%$ & $100.0 \%$ \\
\hline
\end{tabular}

$\chi^{2}=5.702, d f=4, p=.223$ 


\begin{tabular}{|c|c|c|c|c|c|c|}
\hline & & & \multicolumn{3}{|c|}{ Ethnicity } & \multirow[b]{2}{*}{ Total } \\
\hline & & & Garo & Santal & Bengali & \\
\hline \multirow[t]{6}{*}{ Ever severe sexual severity } & \multirow[t]{3}{*}{ None } & Count & 318 & 319 & 322 & 959 \\
\hline & & $\%$ within Ethnicity & $100.0 \%$ & $100.0 \%$ & $99.7 \%$ & $99.9 \%$ \\
\hline & & Standardized Residual & .0 & .0 & .0 & \\
\hline & \multirow[t]{3}{*}{ Mild } & Count & 0 & 0 & 1 & 1 \\
\hline & & \% within Ethnicity & $0.0 \%$ & $0.0 \%$ & $0.3 \%$ & $0.1 \%$ \\
\hline & & Standardized Residual & -.6 & -.6 & 1.1 & \\
\hline \multirow{2}{*}{\multicolumn{2}{|c|}{ Total }} & Count & 318 & 319 & 323 & 960 \\
\hline & & \% within Ethnicity & $100.0 \%$ & $100.0 \%$ & $100.0 \%$ & $100.0 \%$ \\
\hline
\end{tabular}

$\chi^{2}=1.974, d f=2, p=.373$

\begin{tabular}{|c|c|c|c|c|c|c|}
\hline & & & \multicolumn{3}{|c|}{ Ethnicity } & \multirow[b]{2}{*}{ Total } \\
\hline & & & Garo & Santal & Bengali & \\
\hline \multirow[t]{6}{*}{ Ever marital any abuse } & \multirow[t]{3}{*}{ No } & Count & 266 & 305 & 294 & 865 \\
\hline & & $\%$ within Ethnicity & $83.6 \%$ & $95.6 \%$ & $91.0 \%$ & $90.1 \%$ \\
\hline & & Standardized Residual & -1.2 & 1.0 & .2 & \\
\hline & \multirow[t]{3}{*}{ Yes } & Count & 52 & 14 & 29 & 95 \\
\hline & & $\%$ within Ethnicity & $16.4 \%$ & $4.4 \%$ & $9.0 \%$ & $9.9 \%$ \\
\hline & & Standardized Residual & 3.7 & -3.1 & -.5 & \\
\hline \multirow{2}{*}{\multicolumn{2}{|c|}{ Total }} & Count & 318 & 319 & 323 & 960 \\
\hline & & $\%$ within Ethnicity & $100.0 \%$ & $100.0 \%$ & $100.0 \%$ & $100.0 \%$ \\
\hline
\end{tabular}

$\chi^{2}=26.022, \mathrm{df}=2, \mathrm{p}<.001$

\begin{tabular}{|c|c|c|c|c|c|c|}
\hline & & & & Ethnicity & & \\
\hline & & & Garo & Santal & Bengali & Total \\
\hline Ever marital poly abuse & No & Count & 318 & 319 & 323 & 960 \\
\hline & & $\%$ within Ethnicity & $100.0 \%$ & $100.0 \%$ & $100.0 \%$ & $100.0 \%$ \\
\hline & & Standardized Residual & .0 & .0 & .0 & \\
\hline Total & & Count & 318 & 319 & 323 & 960 \\
\hline & & $\%$ within Ethnicity & $100.0 \%$ & $100.0 \%$ & $100.0 \%$ & $100.0 \%$ \\
\hline
\end{tabular}

